\title{
Polypropylene Crystallization as a Physical Gelation Process
}

\author{
Natalia V. Pogodina and H. Henning Winter* \\ Department of Chemical Engineering and Department of Polymer Science and Engineering, \\ University of Massachusetts, Amherst, Massachusetts 01003 \\ Received J anuary 30, 1998; Revised Manuscript Received August 7, 1998
}

\begin{abstract}
Early stages of crystallization of polymers may be viewed as physical gelation. This is shown with four commercial isotactic polypropylenes, which have been studied by dynamic mechanical experiments at low degrees of undercooling, $\Delta T=10-26 \mathrm{~K}$, below their nominal melting temperature. The physical gel point is manifested by slow power law dynamics, which expresses itself in a shear relaxation modulus $\mathrm{G}(\mathrm{t})=\mathrm{St} \mathrm{t}^{-\mathrm{n}}$ at long times, $\lambda_{0}<\mathrm{t}<\lambda_{\mathrm{pg}}$, where $\mathrm{S}$ is the gel stiffness, $\mathrm{n}$ is the relaxation exponent, $\lambda_{0}$ is the crossover to short time dynamics (entanglements, gl ass modes), and $\lambda_{\mathrm{pg}}$ is the longest relaxation time, which can be considered to be infinite for our experiments due to the long lifetime of the physical bonds. The time to reach the gel point (gel time $t_{c}$ ) decreases exponentially with $\Delta T$, and the critical gel becomes stiffer (smaller $\mathrm{n}$, larger $\mathrm{S}$ ) with increasing $\Delta \mathrm{T}$. The absolute critical crystallinity at the gel point, $X_{c}$, was found to be only about $2 \%$ or less. This value was determined from published DSC data which, however, needed to be extrapolated to $t_{c}$, as measured by mechanical spectroscopy. This very low crystallinity suggests that only a few junctions are necessary to form a sample spanning network. The network in this case is "loosely" connected, and the critical gel is soft.
\end{abstract}

\section{Introduction}

Rheological properties of semicrystalline polymers respond very sensitively al ready to the early stages of crystallization. At these early stages, crystallization may be viewed as a physical gelation process. ${ }^{1-5}$ Crystalline order slows down the molecular motions while increasing the connectivity between molecules. Motions of groups of molecules correlate over considerable distance (growing correlation length). The state at which the correlation length diverges may be defined as the physical gel point (GP). Surprisingly, this gel point occurs at very low crystallinity, as shown by Schwittay et al. ${ }^{1}$ on isotactic polypropylene (iPP). The crystallinity at the gel point, $X_{c}$, was found to be about $3 \%$ at slow crystallization and slightly higher at increased rate of crystallization (higher undercooling, $\Delta \mathrm{T}$ ). The observations of Schwittay et al. ${ }^{1}$ were confirmed and, possibly, explained by recent experiments of Akpalu et al. ${ }^{6}$ who, for a crystallizing polyethylene (PE), found that spherulites reach their full size after very short times of crystallization and at very low crystallinity. This similarity in behavior is quite surprising in view of the very different crystal habit in PE and iPP.

Early stages of crystallization in iPP were also studied by Okada et al. ${ }^{7}$ who found that a disordered crystalline domain with low crystallinity is formed at early stages. However, the experiment was performed at considerably faster kinetics of crystallization due to the high degree of undercooling $\Delta \mathrm{T}$. Slow crystallization kinetics in iPP was studied by Olley et al. ${ }^{8}$ The important result was that at very low undercooling (i.e., slow crystallization) the first growing objects are anisometric sheaf-like aggregates that exhibit the dominant direction of growth. With the increase of undercooling, randomization in space of the orientation of the growth direction occurs. As a result, at lower crystallization temperatures the growing objects round out to spheruilte structures more rapidly and spherulites have a smaller size at their final stage.

All our experiments concern small $\Delta T$. In comparison, thermally activated processes are commonly ex- pressed by exponential functions with variable temperature parameter

$$
\frac{1}{T_{\exp }}-\frac{1}{T_{m}}=\frac{T_{m}-T_{\exp }}{T_{m} T_{\exp }} \approx \frac{\Delta T}{T_{m}^{2}}
$$

where $T_{m}$ is the nominal melting temperature and $T_{\exp }$ is the temperature of the isothermal crystallization experiment. As the temperature range in our experiment is narrow, i.e., $T_{\exp } \approx T_{m}, \Delta T$ can be used as an independent parameter for the physical gelation. Another temperature parameter $\left(1 / T_{\exp } \Delta T\right)$ is commonly used in the crystallization literature. ${ }^{9,10}$

Compared to chemically cross-linking systems, it is more difficult to define the gel point for physical gels. While the chemical gel point is defined by a diverging molecular weight $M_{w}$, the molecular weight of molecules in physical gels does not change when the supramolecular clusters form (by crystallization, for instance). Therefore, standard dissolution methods of chemical gelation cannot be applied to detect the state of a physical gelation process or to detect the divergence of physical cluster size at the gel point. Instead of that, we use the evolving relaxation time spectrum and assume universality for the rheological behavior at the gel point. The infinite network at the gel point is manifested in a strong coupling of relaxation modes over a wide range of size scales. It results in the slow power law dynamics of the CW relaxation time spectrum ${ }^{11-13}$

$$
\mathrm{H}(\lambda)=\mathrm{S} \frac{\lambda^{-\mathrm{n}}}{\Gamma(\mathrm{n})} \quad \text { for } \quad \lambda_{0}<\lambda<\lambda_{\mathrm{pg}}
$$

and in a power law relaxation modulus in the terminal zone

$$
\mathrm{G}(\mathrm{t})=\mathrm{St}^{-\mathrm{n}} \quad \text { for } \quad \lambda_{0}<\mathrm{t}<\lambda_{\mathrm{pg}}
$$


Table 1. Molecular and Rheological Parameters of Isotactic Polypropylenes in Supercooled Melts (Reference Temperature is $T_{\text {ref }}=140{ }^{\circ} \mathrm{C}$ )

\begin{tabular}{lllll}
\hline sample & \multicolumn{1}{c}{ A } & \multicolumn{1}{c}{ B } & \multicolumn{1}{c}{ C } \\
\hline $\mathrm{M}_{\mathrm{n}}(\mathrm{g} / \mathrm{mol})$ & $84.168 \times 10^{3}$ & $96.847 \times 10^{3}$ & $71.304 \times 10^{3}$ & $72.975 \times 10^{3}$ \\
$\mathrm{M}_{\mathrm{w}}(\mathrm{g} / \mathrm{mol})$ & $351.163 \times 10^{3}$ & $344.134 \times 10^{3}$ & $386.556 \times 10^{3}$ & $337.251 \times 10^{3}$ \\
$\mathrm{M}_{\mathrm{z}}(\mathrm{g} / \mathrm{mol})$ & $1004.781 \times 10^{3}$ & $907.365 \times 10^{3}$ & $1199.787 \times 10^{3}$ & $963.576 \times 10^{3}$ \\
$\mathrm{M}_{\mathrm{w}} / \mathrm{M}_{\mathrm{n}}$ & 4.17 & 3.55 & 5.42 & 4.62 \\
$\mathrm{M}_{\mathrm{z}} / \mathrm{M}_{\mathrm{w}}$ & 2.86 & 2.64 & 3.10 & 2.86 \\
$\omega_{\times}(\mathrm{rad} / \mathrm{s})$ & 2.1 & 20 & 1.05 & 2.6 \\
$\mathrm{G}^{\prime}=\mathrm{G}^{\prime \prime}{ }_{x}(\mathrm{~Pa})$ & $3 \times 10^{4}$ & $3 \times 10^{4}$ & $1.4 \times 10^{4}$ & $2 \times 10^{4}$ \\
$\eta_{0}(\mathrm{~Pa} \mathrm{~s})$ & $6 \times 10^{4}$ & $4 \times 10^{4}$ & $5 \times 10^{4}$ & $5 \times 10^{4}$ \\
$\mathrm{G}_{0}(\mathrm{~Pa})$ & $1 \times 10^{5}$ & $1 \times 10^{5}$ & $1 \times 10^{5}$ & $1 \times 10^{5}$
\end{tabular}

$\mathrm{S}$ is the gel stiffness, $\mathrm{n}$ is the critical relaxation exponent, $\lambda$ is the relaxation time, and $\Gamma(n)$ is the gamma function. $\lambda_{0}$ denotes the crossover to some faster dynamics that depends on the molecular detail on a smaller scale. Here we apply the concepts and equations of chemical gelation, ${ }^{11-13}$ eqs 2 and 3 , to physical gelation. The upper cutoff time of the physical gel, $\lambda_{\mathrm{pg}}$, is the finite lifetime of the largest cluster. ${ }^{14}$

In oscillatory shear at the gel point, the dynamic moduli scale with frequency

$$
\begin{aligned}
& \mathrm{G}^{\prime}{ }_{\mathrm{c}}=\frac{\mathrm{G}^{\prime \prime}{ }_{\mathrm{c}}}{\tan \delta}=\mathrm{S} \Gamma(1-\mathrm{n}) \cos \frac{\mathrm{n} \pi}{2} \omega^{\mathrm{n}} \quad \text { for } \\
& 1 / \lambda_{\text {pg }}<\omega<1 / \lambda_{0}
\end{aligned}
$$

A consequence of power-law dynamics is independence of the loss tangent $\tan \delta$ of frequency at the gel point:

$$
\begin{array}{ll}
\tan \delta_{\mathrm{c}}=\frac{\mathrm{G}^{\prime \prime}{ }_{\mathrm{c}}}{\mathrm{G}^{\prime}{ }_{\mathrm{c}}}=\tan \frac{\mathrm{n} \pi}{2}=\mathrm{const} & \text { for } \\
& 1 / \lambda_{\mathrm{pg}}<\omega<1 / \lambda_{0}
\end{array}
$$

that provides the most reliable and generally valid method to determine the gel time and parameters $n$ and $\mathrm{S}$ of the critical gel. ${ }^{15}$

Chemical critical gels are characterized by a powerlaw distribution of molar mass ${ }^{16}$

$$
N(M) \propto M^{-\tau}
$$

where the exponent value is predicted as $\tau=2.2$ or 2.5 depending on the theoretical approach. ${ }^{17,18}$ Similarly, a broad distribution of physical clusters is expected in the crystallizing polymer. Small angle light scattering experiments are in progress for determining the size and polarizability of the growing clusters during early stages of crystallization.

The crystallization of isotactic polypropylene (iPP) has been studied extensively. 7,8,19-29 Crystal growth in iPP may be viewed as a three-stage process: ${ }^{22-24}$ first stage (primary crystallization), fast growth of spherulites in fibrillar stems; second stage (secondary crystallization), subsequent crystallization of impure interfibrillar melt; third stage, slow crystallization of entangled molecules in the narrow layers of the residual melt. Polypropylene is also known as a morphologically complex polymer, showing several different morphologies dependent on crystallization conditions ${ }^{25-28}$ and a very special form of crystal branching. These branches form approximately normal to the linear growth direction of the lamellae and tend to proliferate in certain temperature ranges. ${ }^{8,25-28}$ At low undercooling, as used in our experiments with a temperature range $140{ }^{\circ} \mathrm{C}<\mathrm{T}_{\exp }<$ $152{ }^{\circ} \mathrm{C}$, iPP crystallizes in its $\alpha$-form. 8,27 The reversible nature of crystalline "junctions" opens the possibility to control the rate of bond formation and the growth rate of the correlation length in solidifying iPP by changing crystallization temperature. Mostly because of its low rate of crystallization iPP is a good model for studying LST as a critical phenomenon.

The present work focuses on the early stages of crystallization at low undercooling (slow crystallization kinetics) of four commercial isotactic polypropylenes and is a continuation of our first investigation. ${ }^{1}$ Emphasis is made on finding a correlation between kinetic characteristics (gel times, crystallization rates) and structural parameters of the critical gel (gel stiffness, relaxation exponent, arystallinity). Corresponding arystallinity data are taken from the literature. ${ }^{29}$

\section{Experimental Section}

Materials. We studied four commercial isotactic polypropylenes (iPP): A (Exxon $4152 \mathrm{E1}$ ), B (Homopolymer from Fina), C (Amoco 9117), D (Phillips FM 95B). No nucleating agent was added. Mol ecular weights and polydispersity of the samples were measured using gel permeation chromatography (GPC) at the Polymer Science Laboratory of the Baytown Polymer Center, Exxon Chemical, Baytown, TX. The samples were of a high molecular weight and high polydispersity (Table 1). A nominal melting temperature of $\mathrm{T}_{\text {melt }}=163^{\circ} \mathrm{C}$ for all four samples was determined as the peak in the DSC (second heating curve with a constant rate $10 \mathrm{~K} / \mathrm{min}$ ). The ideal equilibrium melting temperature for iPP has been reported as $\mathrm{T}_{\mathrm{m}}^{\circ}=208{ }^{\circ} \mathrm{C} .30,31$

Samples were prepared by molding polypropylene pellets for $10 \mathrm{~min}$ at $200{ }^{\circ} \mathrm{C}$ under vacuum in a Carver laboratory press. The samples were then allowed to cool slowly, still under vacuum. Molded samples were stored in a vacuum oven at about $120^{\circ} \mathrm{C}$ to prevent moisture absorption. For rheological studies, samples were melted in the rheometer at 210 ${ }^{\circ} \mathrm{C}$ for $20 \mathrm{~min}$ under nitrogen and then cooled to the experimental temperature, still in a nitrogen atmosphere. From here on throughout the crystallization experiment, the temperature was kept constant at $\mathrm{T}=163^{\circ} \mathrm{C}-\Delta \mathrm{T}$, where the degree of undercooling $\Delta \mathrm{T}$ is an adjustable parameter, $10 \mathrm{~K}<\Delta \mathrm{T}<26$ $\mathrm{K}$.

Rheological Experiment. Small amplitude oscillatory shear (SAOS) was started as soon as the experimental temperature was reached. Cyclic frequency sweeps (CFS) were performed repeatedly in the frequency window between 0.01 and $10 \mathrm{rad} / \mathrm{s}$ ( 5 points per decade). The initial strain of 0.1 (ensuring sufficiently high torque values) was manually decreased during crystallization to stay within the linear viscoelastic region and below the maximum torque of the instrument.

The rheological experiments were performed using the timeresolved rheometry technique ${ }^{32}$ with the advanced rheometric expansion system (ARES) of Rheometric Scientific Inc. (RSI), equipped with parallel plates (diameter $25 \mathrm{~mm}$ ). The instrument is operated by using the RSI Orchestrator, version 6.3.0. The experimental data were analyzed using IRIS and GELPRO software (http://members.tripod.com/ Rheology/). U sing GELPRO, the CFS data are split into separate sets (one for 


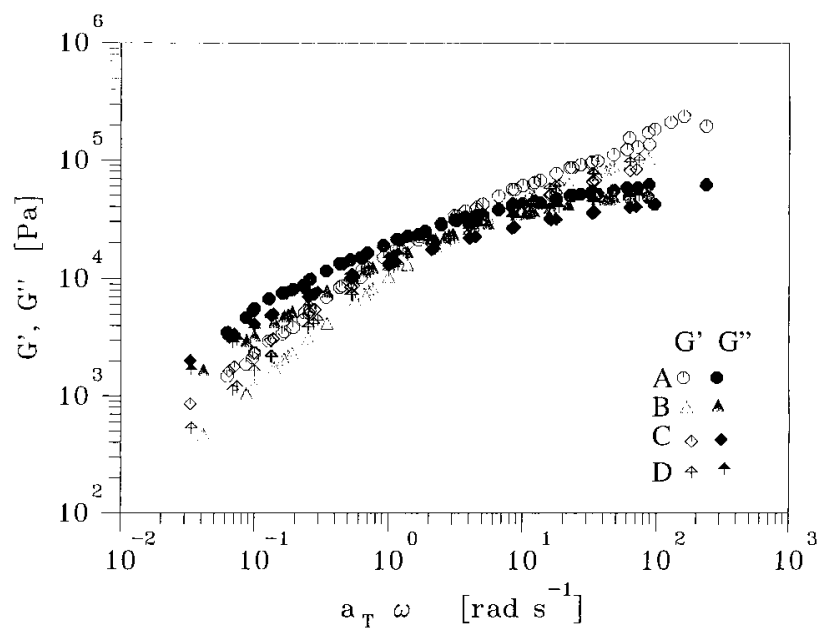

Figure 1. Storage modulus and loss modulus master curves of four isotactic polypropylenes at $\mathrm{T}_{\text {ref }}=140^{\circ} \mathrm{C}$. The frequency sweep data are taken at temperatures 180, 170, 160, 150, 145, 140, and $137^{\circ} \mathrm{C}$. Letters A-D depict the four different iPP samples.

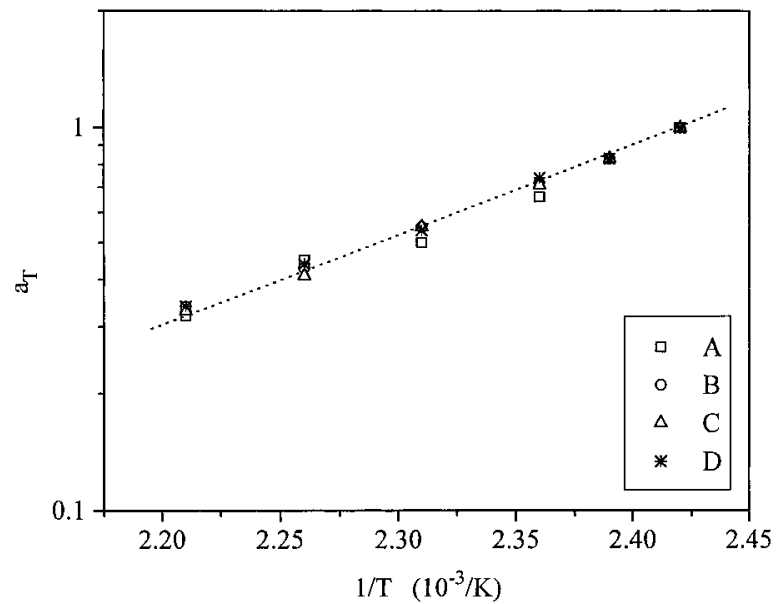

Figure 2. Horizontal temperature shift factor at $T_{\text {ref }}=140$ ${ }^{\circ} \mathrm{C}$ versus inverse temperature.

each experimental frequency) to follow the evolution with time. Frequency-dependent data can then be interpolated at any given time of the experiment, i.e., at any stage of crystallization, resulting in "snapshots" of intermediate rheological patterns of the crystallizing material.

\section{Experimental Results}

Linear Viscoelasticity of the Melt. G' and $G^{\prime \prime}$ data were shifted to a reference temperature of $\mathrm{T}_{\text {ref }}=140$ ${ }^{\circ} \mathrm{C}$ using time-temperature superposition, ${ }^{33}$ as shown in Figure 1. All data belong to the melt behavior of the polypropylenes, since crystallization is very slow under these conditions. Note that experiments below $\mathrm{T}_{\mathrm{m}}=$ $163^{\circ} \mathrm{C}$ were taken immediately after cooling from 210 ${ }^{\circ} \mathrm{C}$ so that the degree of crystallization was too low yet to affect the rheological properties during a short time in the undercooled state $(\sim 10 \mathrm{~min})$. The rheological behavior of four polypropylene samples in the temperature range $140^{\circ} \mathrm{C}<\mathrm{T}_{\exp }<180^{\circ} \mathrm{C}$ is similar. Horizontal shift factors, $a_{\mathrm{T}}$, follow the Arrhenius law with about the same activation energy (see Figure 2). Vertical shift factors, $b_{T}$, are close to unity. $\eta_{0}$ and $\mathrm{G}_{\mathrm{N}}{ }^{\circ}$ values are presented for all samples in Table 1 and will be used as reference values for later comparison with the

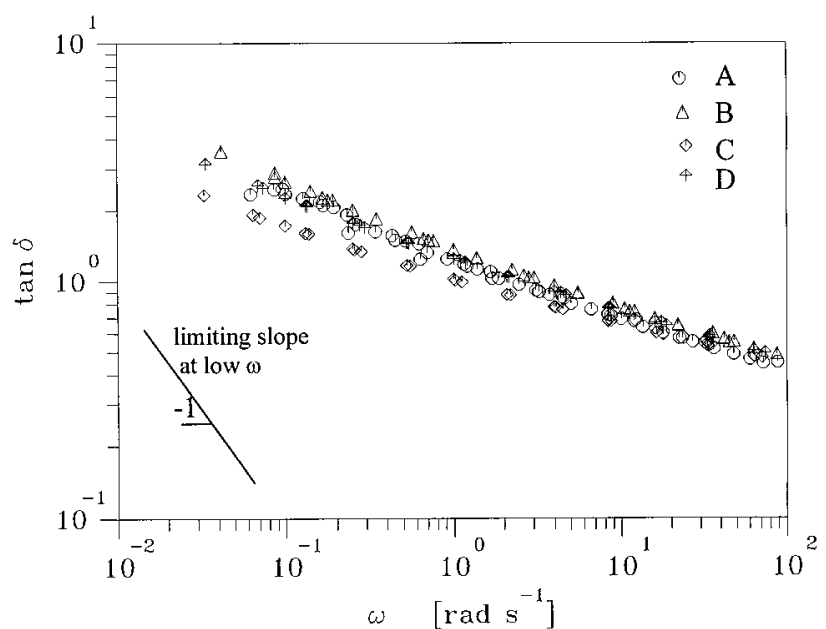

Figure 3. $\tan \delta$ versus frequency $\omega$ for iPP. The limiting slope of minus 1 at low $\omega$ is shown for comparison. This lowfrequency range was not accessible because of the broad crossover between the entanglement and flow regimes (measure of broad molecular weight distribution).

stiffness of the critical gel. Zero shear viscosity values were estimated from the complex dynamic viscosity, where it levels off at low frequencies $\eta_{0} \cong \eta^{*}$ (at $\omega=5$ $\times 10^{-2} \mathrm{rad} / \mathrm{s}$ ). The plateau modulus was taken as the storage modulus where it levels off at high frequencies $\mathrm{G}_{0} \cong \mathrm{G}^{\prime}\left(\right.$ at $\left.\omega=10^{2} \mathrm{rad} / \mathrm{s}\right)$.

For molten iPP, at temperatures above the nominal melting point, the intersect of $\mathrm{G}^{\prime}(\omega)$ and $\mathrm{G}^{\prime \prime}(\omega)$ curves and the shape of $\tan \delta(\omega)$ have been used to characterize the molecular weight distribution and molecular weight values. $^{34,35}$ According to Zeichner and Patel, ${ }^{34}$ the inverse values of the dynamic moduli at their intersect $\mathrm{G}_{x}{ }_{x}=\mathrm{G}^{\prime \prime}{ }_{x}$ characterizes the polydispersity of the samples.

According to that, the close $G^{\prime}{ }_{x}=G^{\prime \prime}{ }_{x}$ values for the four sampl es suggest similar polydispersity for all four. Another rheological method for estimating polydispersity is based on the analysis of the loss tangent $\tan \delta$ versus $\eta_{0} \omega .^{35}$ According to this approach, the slope in the $\tan \delta$ vs $\eta_{0} \omega$ plot can be regarded as a measure of the width of the molecular weight distribution (MWD). Figure 3 shows such plots of $\tan \delta$ vs $\omega$ for our samples ( $\eta_{0}$ is practically the same for our samples and will result only in a horizontal shift, but will not affect the slope); the slope varies in the range $0.28-0.42$. This might be considered as a qualitative sign of similar and broad MWD for all four samples.

Dynamics of Solidification (by Crystallization). During isothermal crystallization at a temperature $T_{\text {exp }}$ $=\mathrm{T}_{\mathrm{m}}-\Delta \mathrm{T}$, the dynamic moduli at different frequencies evolve gradually with time. This is shown for $\mathrm{T}_{\exp }=$ $150{ }^{\circ} \mathrm{C}$ in Figure 4. After a long induction period, $\mathrm{G}^{\prime}$ and $G^{\prime \prime}$ begin to grow strongly and soon $G^{\prime}$ exceeds $G^{\prime \prime}$. At the end, $\mathrm{G}^{\prime}$ dominates the experiment. The growth of relative crystallinity in time (in terms of the Avrami model; seealso Discussion) is plotted on the same graph. One can see how little crystallinity is devel oped compared to dynamic moduli. The gel point (GP) can be extracted by presenting the data in terms of the loss tangent $\tan \delta$ and looking for the frequency independence of slow power law dynamics. Figure 5 shows the plot of $\tan \delta$ versus frequency at interpolated times. The "flat" phase angle region covers a wide frequency window, as marked by a horizontal line. Only the lowfrequency "flat" region is associated with the gel point. 


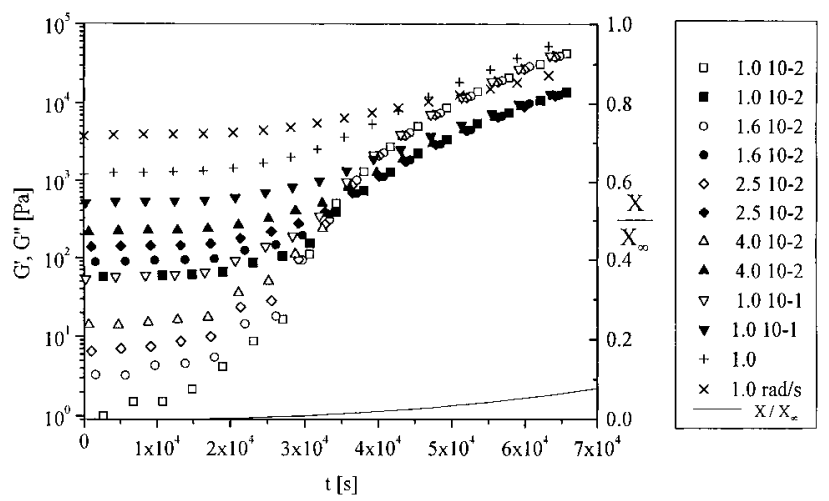

Figure 4. Growth of dynamic moduli G', and G" during cyclic frequency sweep for the sample $B$ (left $Y$-axis) and relative crystallinity according to Avrami eq 12 and DSC data by Kim et al. (right $\mathrm{Y}$-axis) at a crystallization temperature of $\mathrm{T}_{\exp }=$ $150{ }^{\circ} \mathrm{C}$. Different symbols depict different frequencies in the frequency range $\omega=10^{-2}-1 \mathrm{rad} / \mathrm{s}$. Open symbols and crosses $(+)$ represent $G^{\prime}$, filled symbols and $(x)$ represent $G^{\prime \prime}$. The corresponding growth of relative crystallinity in time (from literary data ${ }^{29}$ ) is represented by a line.

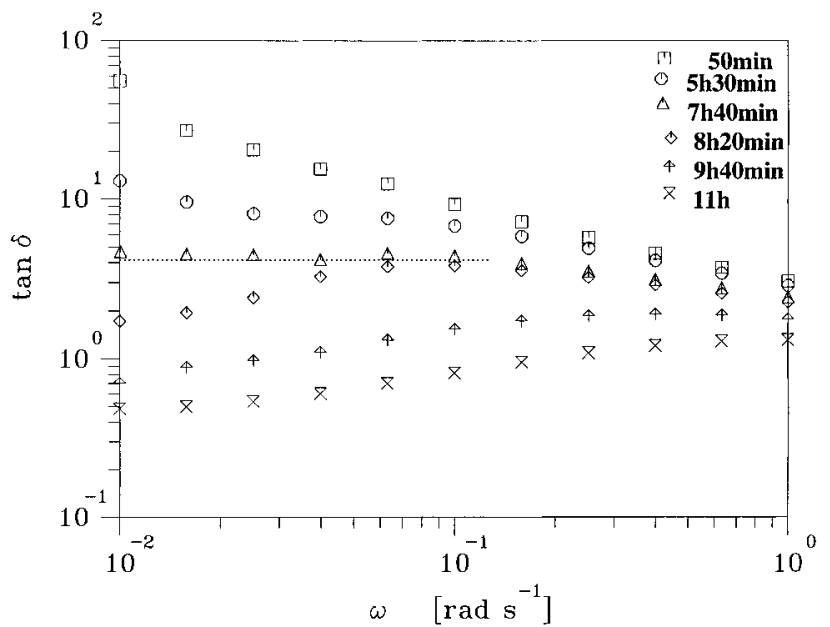

Figure 5. Interpolated tan $\delta$ versus frequency at different instances during the crystallization process $\left(\mathrm{T}_{\exp }=150^{\circ} \mathrm{C}\right)$ for sample B. Different symbols depict the interpolation times given in the figure. The estimated gel time is $7 \mathrm{~h} 40 \mathrm{~min}$.

When working at low frequencies (i.e., long experimental times), one has to be sure that criteria of slow mutation are met:32,36,37 the rate of crystallization has to be slow enough so that the continuous change of modulus can be considered negl igible during the taking of single data points (experimental time of about $\Delta t=$ $2 \pi / \omega)$. This is ensured by a mutation number

$$
\mathrm{N}_{\mathrm{mu}}=\frac{\Delta \mathrm{t}}{\lambda_{\mathrm{mu}}}=\left(\frac{2 \pi}{\omega}\right)\left(\frac{1}{\mathrm{G}^{\prime}}\right)\left(\frac{\partial \mathrm{G}^{\prime}}{\partial \mathrm{t}}\right)
$$

which is sufficiently small, $\mathrm{N}_{\mathrm{mu}} \ll 1$. The mutation time $\lambda_{\mathrm{mu}}=\left[\left(1 / \mathrm{G}^{\prime}\right)(\mathrm{dG} / \mathrm{dt})\right]^{-1}$ is equal to the inverse of the $\mathrm{G}^{\prime}$ slope in Figure 4. We chose to define this time for the change in the rheol ogical val ue with the storage modulus $\mathrm{G}^{\prime}$ since it changes faster than $\mathrm{G}^{\prime \prime}$. The mutation numbers for $G^{\prime \prime}$ were less than half of the values determined for the corresponding $\mathrm{G}^{\prime}$.

The above condition was checked for each individual data point in our study and gave mutation number values $\mathrm{N}_{\mathrm{mu}} \leq 0.3$ for $\mathrm{G}^{\prime}$ at the lowest frequency $\omega=$ $10^{-2} \mathrm{rad} / \mathrm{s}$ and at $\mathrm{T}_{\exp }=145^{\circ} \mathrm{C}$. At lower temperatures $138^{\circ} \mathrm{C}<\mathrm{T}_{\exp }<145^{\circ} \mathrm{C}$ the lowest accessible frequency

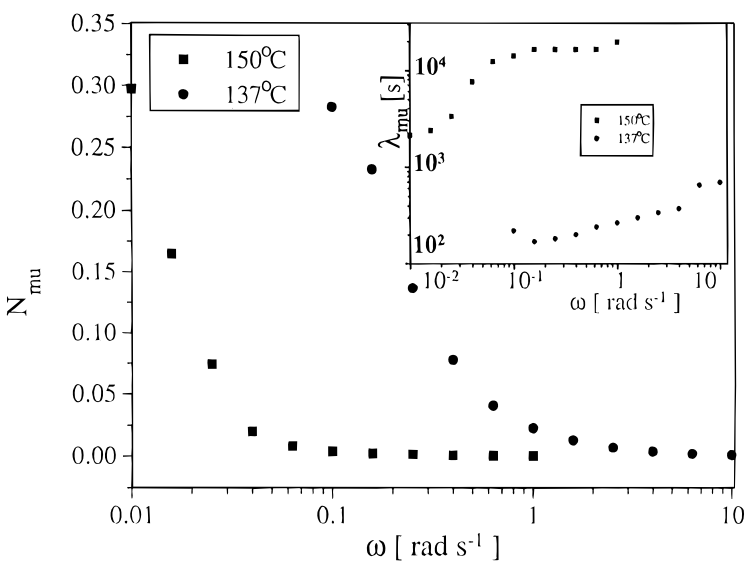

Figure 6. Mutation number versus frequency at two different temperatures depicted on the figure. The inset has corresponding mutation times plotted versus frequency for the same temperatures.

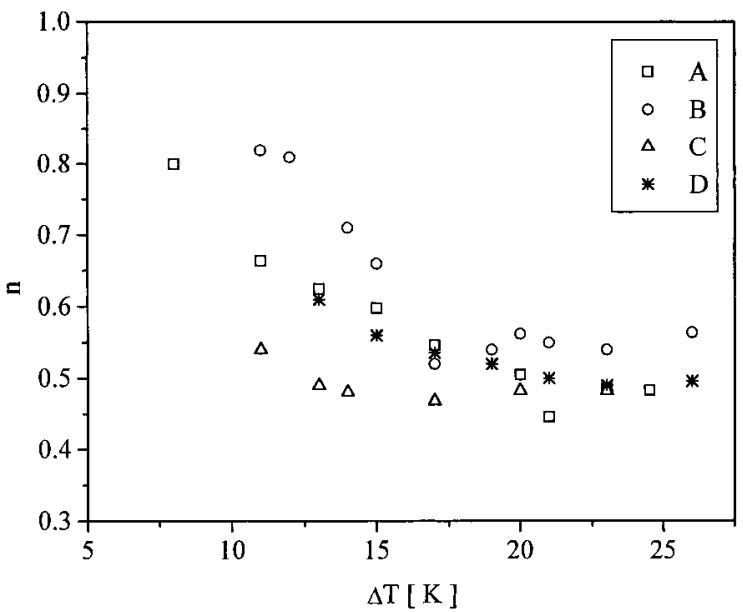

Figure 7. Relaxation exponent $\mathrm{n}$ versus the degree of undercooling for the four isopropylene samples depicted in the picture. Relaxation exponents are evaluated at the gel point according to eqs 4 , and 5.

had to be increased to $\omega=10^{-1} \mathrm{rad} / \mathrm{s}$ in order to maintain a low mutation number (see Figure 6). Samples with $\mathrm{N}_{\mathrm{mu}} \leq 0.3$ are treated as quasi-stable during individual measurements and rheological parameters are considered to be meaningful. Gel times at all crystallization temperatures were determined in a similar way (see Figure 6). Mean values of gel stiffness $\mathrm{S}$ and relaxation exponent $\mathrm{n}$ as calculated from eqs 4 , and 5 at $\mathrm{G}^{\prime}=\mathrm{G}^{\prime}{ }_{c}$ will be discussed in the next section.

Rheological Properties of the Critical Gels. Figures 7 and 8 show measured $n$ and $S$ values vs the degree of undercooling $\Delta T=T_{m}-T_{\text {exp. }}$. The general trends are the same for all four critical gels: (1) at higher undercooling, $\Delta \mathrm{T}$, iPP samples are characterized by a relaxation exponent value of $\mathrm{n} \approx 0.5$ and gel stiffness $\mathrm{S} \approx 2 \times 10^{4} \mathrm{~Pa} \mathrm{~s}^{\mathrm{n}} ; 2$ ) at low undercooling $\Delta \mathrm{T}$, critical gels become "softer" and more fragile, which is reflected in the increase in $n$ values (Figure 7 ) with the simultaneous decrease in $\mathrm{S}$ values (Figure 8). Moreover, $\mathrm{n}$ and $\mathrm{S}$ values differ strongly from sample to sample at low $\Delta T$. That gives the possibility to distinguish them from each other according to their "soft" or "stiff" behavior. Sample B has the most "soft" critical gel (at $\Delta T=10 \mathrm{~K}, \mathrm{n}=0.8, \mathrm{~S}=5 \times 10^{3} \mathrm{~Pa}^{\mathrm{n}}$ ) followed by sample $A$ (at $\Delta T=10 \mathrm{~K}, \mathrm{n}=0.65, \mathrm{~S}=7.5 \times 10^{3} \mathrm{~Pa}$ 


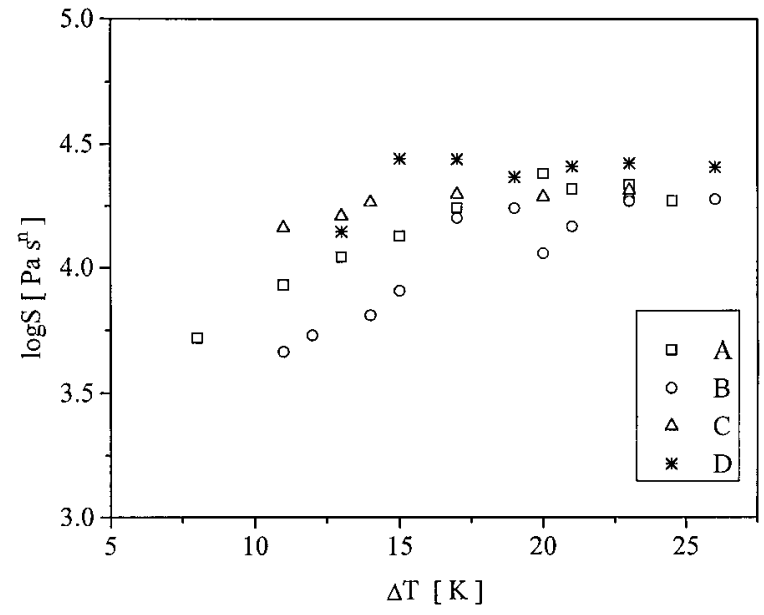

Figure 8. Gel stiffness $S$ versus the degree of undercooling for the four isopropylene samples A-D. Front factors are estimated at the gel point according to eqs 4 , and 5 .

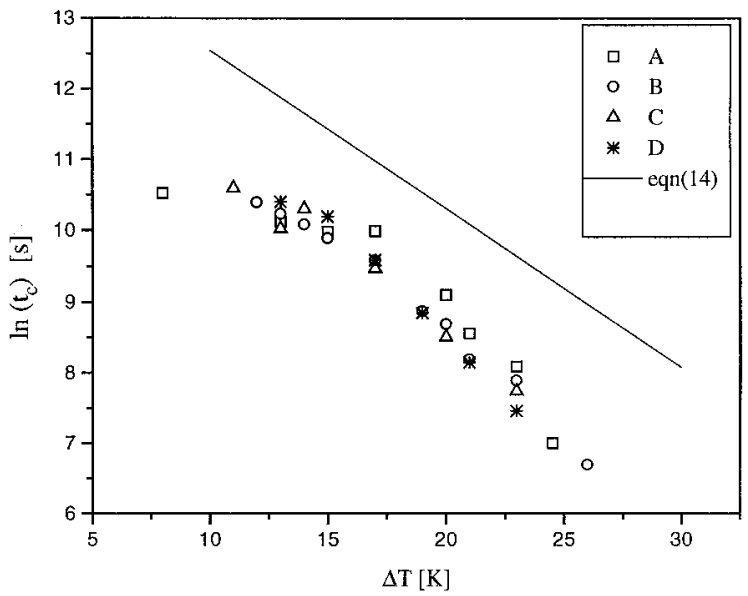

Figure 9. Semilogarithmic plot of critical gel times $t_{c}$ versus degree of undercooling for the four polypropylene samples $A-D$. The solid line represents Avrami times $t_{A}(\Delta T)$ estimated according to eq 14, which will be explained below in the Discussion.

$\mathrm{s}^{\mathrm{n}}$ ). Samples $C$ and D show the most "stiff" behavior: at $\Delta \mathrm{T}=10 \mathrm{~K}, \mathrm{n}=0.55, \mathrm{~S}=1.5 \times 10^{4} \mathrm{~Pa} \mathrm{~s}^{\mathrm{n}}$.

The logarithm of the critical gel time $\ln _{\mathrm{c}}$ (taken from the low-frequency condition $\tan \delta=$ const) is plotted versus the degree of undercooling in Figure 9. The data may be approximated by a simple exponential decay ${ }^{1}$

$$
t_{c}=a e^{-b \Delta T}
$$

We will use this relation for a linear analysis of the data (see eq 15 below).

Thermodynamically, $\Delta \mathrm{T}$ may be interpreted as the driving force of crystallization, large values resulting in a short crystallization time; $b$ is inverse proportional to some activation energy, normalized by the universal gas constant R. Fit parameters $a$ and $b$ are presented in Table 2. One can notice significant differences in a and $b$ parameters, which reflect the differences in structural details in the four samples.

\section{Discussion}

Strain Effect on Crystallization Behavior. Stress and strain are known to affect crystallization behavior
Table 2. Coefficients of Linear Regression ( $E q$ 8) and Extremum Condition (Eq 19) (Using A $=2.66 \times 10^{6} \mathrm{~s}$, B $=$ $0.224 \mathrm{~K}^{-1}$, and $\mathrm{N}=2.5$ As Extrapolated from Data of Kim et al. ${ }^{29}$ )

\begin{tabular}{ccccl}
\hline sample & $10^{-6} \mathrm{a}(\mathrm{s})$ & $\mathrm{b}\left(\mathrm{K}^{-1}\right)$ & $\mathrm{B} / \mathrm{b}$ & $\mathrm{A} / \mathrm{a}$ \\
\hline $\mathrm{A}$ & 0.33 & 0.2 & $1.13>1$ & 8.0 \\
$\mathrm{~B}$ & 0.91 & 0.26 & $0.86<1$ & 2.92 \\
$\mathrm{C}$ & 0.64 & 0.24 & $0.93<1$ & 4.16 \\
$\mathrm{D}$ & 2.32 & 0.31 & $0.72<1$ & 1.15
\end{tabular}

in terms of its kinetics as well as in the mechanism of nucleation. ${ }^{38-42}$ However, our results on crystallization were obtained at low strains $0.01-0.03$. An initial strain of 0.1 was used only to monitor the mechanical behavior in the melt. Figure 9 shows that gel times measured in the range of strain $0.01-0.03$ do not differ from each other. This indicates that such low strains do not affect nucleation and crystallization behavior significantly. We are in the process of including large strain effects in a newly started study.

Relaxation Exponent and Stiffness of the Critical Gel. The condition used for the determination of the gel time (and consequently for the true $\mathrm{n}$ and $\mathrm{S}$ values) is power law relaxation at sufficiently low frequencies. This can be detected by the "flat" phaseangle region (independence of frequency) in a sufficiently wide frequency range of the terminal zone $(\omega$ $<10^{-1} \mathrm{rad} / \mathrm{s}$ ). However, there are inherent constraints to the experiment that limit the accessible frequency range. Especially, low enough frequencies may not be accessible. In the data analysis, this may lead to an apparently later gel point (systematically higher gel time) and consequently lower $n$ value (see Figure 5). The actual gel point might even occur earlier than what we have reported here. This is why we need to evaluate the lower limit of the experimentally accessible frequency range.

The experimental problems at low frequencies arise from the fact that the experimental time in eq $7, \Delta t=$ $2 \pi / \omega$, grows inversely with $\omega$. In addition, the growth rate of both dynamic moduli increases with ${ }^{43}$

$$
\left(\frac{1}{\mathrm{G}^{\prime}} \frac{\partial \mathrm{G}^{\prime}}{\partial \mathrm{t}}\right)_{\omega} \sim\left(\frac{1}{\mathrm{G}^{\prime \prime}} \frac{\partial \mathrm{G}^{\prime \prime}}{\partial \mathrm{t}}\right)_{\omega} \sim \omega^{-\kappa}
$$

where the exponent $\kappa$ had adopted values of about $\kappa \approx$ 0.2 for all previously studied materials. As a result, the mutation number changes with frequency (see eq 7) as $\mathrm{N}_{\mathrm{mu}} \sim \omega^{\kappa-1}=\omega^{-0.8}$. A decrease in frequency by one decade would increase the mutation number by a factor of 6.3. If the criterion of slow mutation will still be met, i.e., $\mathrm{N}_{\mathrm{mu}}<0.3$, then this shift to lower frequencies is possible. In our case, however, at $\mathrm{T}_{\text {exp }}<145^{\circ} \mathrm{C}$, the accuracy of the gel time measurement cannot be improved by measuring at frequencies lower than $\omega<10^{-1}$ $\mathrm{rad} / \mathrm{s}$, as this would result in large $\mathrm{N}_{\text {mu }}$ values.

The two rheological parameters of the critical gel, relaxation exponent $\mathrm{n}$ and gel stiffness $\mathrm{S}$, are very sensitive characteristics of the polymer. The critical gel can be identified as "soft" or "stiff", depending on the molecular and structural details, reflected in $\mathrm{n}$ and $\mathrm{S}$. The range of experimental $n$ and $S$ values for the $4 \mathrm{iPP}$ samples $0.5<\mathrm{n}<0.8$ and $4 \times 10^{3}\left[\mathrm{~Pa} \mathrm{~s}^{\mathrm{n}}\right]<\mathrm{S}<3 \times$ $10^{4}\left[\mathrm{~Pa} \mathrm{~s}^{\mathrm{n}}\right]$, shows that polypropylenes form critical gels that are extremely soft (small $\mathrm{S}$, large $\mathrm{n}$ ) at low $\Delta \mathrm{T}$ and stiffer at increased $\Delta \mathrm{T}$. 


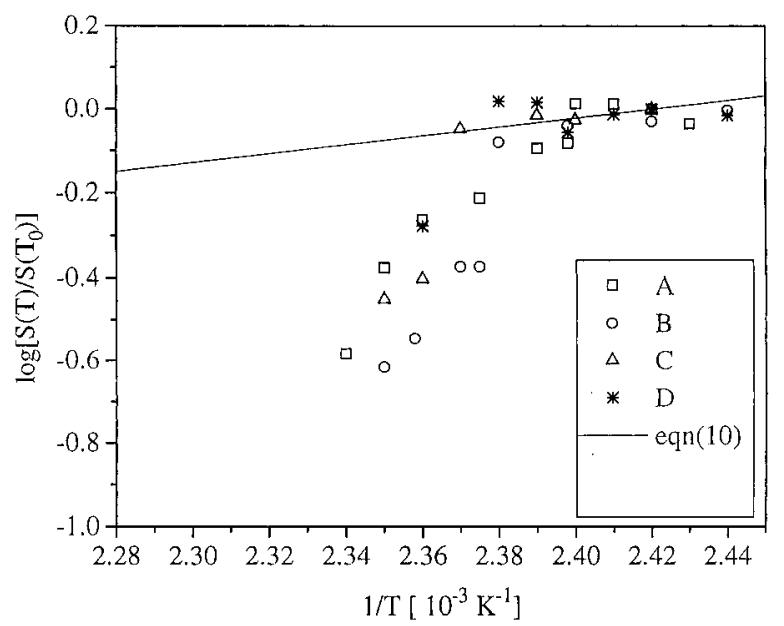

Figure 10. Gel stiffness $S(T)$ normalized to the gel stiffness at a reference temperature $\mathrm{S}\left(\mathrm{T}_{0}\right)$ versus inverse crystallization temperature $\mathrm{T}^{-1}$. The reference temperature is $\mathrm{T}_{\mathrm{o}}=140^{\circ} \mathrm{C}$. Different samples are depicted by different symbols on the figure. The straight line represents eq $10 . \mathrm{a}_{\mathrm{T}}^{\mathrm{m}} / \mathrm{bT}$ with data from Figure 2, and bT set equal to 1 .

Izuka et al. ${ }^{44}$ showed that stiffness $S(T)$ of chemically cross-linked chemical gels shifts with temperature as

$$
\mathrm{S}(\mathrm{T})=\mathrm{S}\left(\mathrm{T}_{0}\right) \frac{\mathrm{a}_{\mathrm{T}} \mathrm{b}_{\mathrm{T}}^{\mathrm{n}}}{\mathrm{b}_{\mathrm{T}}}
$$

where $\mathrm{S}\left(\mathrm{T}_{0}\right)$ is the gel stiffness at reference temperature $T_{0}$. Figure 10 shows the plot of $\log \left[S(T) / S\left(T_{0}\right)\right]$ vs $1 / T$ for iPP-critical gels. Points represent experimental data, and the solid line is calculated according to eq 10. Equation 10 fits experimental data only in the very narrow temperature range close to reference temperature $\left(\mathrm{T}=140{ }^{\circ} \mathrm{C}\right)$. It is obvious that at low crystallization rates the behavior of the gel stiffness cannot be described only in terms of time-temperature superposition. Molecular and structural details of the samples, which strongly affect crystallization processes, are assumed to be significantly different at low and high $\Delta \mathrm{T}$. It would be interesting to explore the structure of the critical gels as functions of $\Delta \mathrm{T}$ (not attempted here).

Scanlan and Winter ${ }^{43}$ and I zuka et al. ${ }^{44,45}$ attempted to relate the critical gel stiffness of chemically crosslinking systems to the properties of the precursor (zero shear viscosity $\eta_{\mathrm{p}}$ ) and the fully cross-linked material (plateau modulus $\mathrm{G}_{0}$ ). They could estimate the stiffness of chemical critical gels with a relation

$$
\mathrm{S}=\mathrm{G}_{0}\left(\frac{\eta_{\mathrm{p}}}{\mathrm{G}_{0}}\right)^{\mathrm{n}}
$$

However eq 11 does not hold for our crystallizing samples. Property S is not independent but it depends on the relaxation exponent for iPP samples in a more complicated way than eq 11 predicts. For the two limiting cases of pure solid and pure liquid, eq 11 predicts for our samples $\log S=\log G_{0}=5.0$ (for $n=0$ ) and $\log S=\log \eta_{p}=4.7$ (for $n=1$ ). Experimental points for the four crystallizing samples are in the range 4.53.7, which is significantly lower than the predicted range 5.0-4.7. New concepts have to be developed to explain critical gel properties of crystallizing polypropylenes.
Kinetics of Crystallization. To get deeper insight into crystallization at its early stages we made an attempt to estimate the crystallinity at the gel point. Crystallinity data any time during crystallization (including the gel point) can be expressed in the Avrami equation $46-48$

$$
\frac{X}{X_{\infty}}=1-e^{-\left(t / t_{A}\right)^{N}}
$$

where the crystallinity $X$ is defined as a fraction of the crystalline phase in the polymer, $t$ is the duration of the crystallization experiment, and $\mathrm{N}$ is the Avrami exponent, which is related to the crystal growth dimension. $\mathrm{X}_{\infty}$ is the maximum crystallinity at long times.

In eq 12, we introduced the concept of an Avrami time $t_{A}$. It is the time at $t=t_{A}$ by which the relative crystallinity

$$
\frac{\mathrm{X}}{\mathrm{X}_{\infty}}=1-\frac{1}{\mathrm{e}}=0.63
$$

has reached $63 \%$ of the final crystallinity $X_{\infty}$. $t_{A}$ is a measure of the time needed to reach the late stages of crystallization.

Kim et al ${ }^{29}$ studied the temperature dependence of iPP crystallization in the absence of crystallization aids. They measured (by DSC) relative crystallinity $X(t)$ of neat iPP at distinct temperatures $T_{\text {exp }}$ in the range $123-$ $136{ }^{\circ} \mathrm{C}$. From their data $\mathrm{X}\left(\mathrm{t}, \mathrm{T}_{\exp }\right), \mathrm{Kim}$ evaluated an isothermal rate constant $\mathrm{K}$ and Avrami exponent $\mathrm{N}$ for specific experimental temperatures $T_{\text {exp. }}$ The data set for K (Kim's results) was converted to $\mathrm{t}_{\mathrm{A}}=\mathrm{K}^{-1 / \mathrm{N}}$ in our study and extrapolated in a $\ln \left(\mathrm{t}_{\mathrm{A}}\right)$ versus $\Delta \mathrm{T}=\mathrm{T}_{\mathrm{m}}-$ $\mathrm{T}_{\text {exp }}$ plot, which leads to a simple approximation

$$
t_{A}=A e^{-B \Delta T}
$$

with $A=2.66 \times 10^{6} \mathrm{~s}$ and $B=0.224 \mathrm{~K}^{-1}$. Since no data at low $\Delta \mathrm{T}$ are available, we base our estimate on the assumption that eq 14 holds for the extended low $\Delta T$ values, i.e., for high experimental temperatures up to $T_{\exp }=153{ }^{\circ} \mathrm{C}$, used in our experiment. We also assume that possible differences in the type of iPP used in Kim's and in our study do not affect eq 14, so that it is still valid for early stages of crystallization of our samples. The Avrami exponent of our samples is set to be $\mathrm{N}=2.5 .^{29}$ The final crystallinity is estimated as $\mathrm{X}_{\infty}$ $=0.6$. Equation 14 with Kim's parameters has al ready been plotted in Figure 9 in order to compare to experimental gel times.

The relative crystallinity was plotted together with $\mathrm{G}^{\prime}$ and $\mathrm{G}^{\prime \prime}$ versus time in Figure 4. One can see how little crystal linity is devel oped at the instant when the gel state is reached. Actual crystallization data cannot be determined from DSC at such low crystallinity (due to the lack of DSC sensitivity). They only can be estimated by extrapolation. Figure 11 shows the relative crystallinity versus relative time $t / t_{A}$ according to eq 12 with the use of Kim's parameter values. Introduction of the Avrami time has the advantage that the conventional Avrami plots at various crystallization temperatures reduce to a single curve (solid line on Figure 11). We define the critical extent of crystallinity as $\mathrm{X}_{\mathrm{c}}=\mathrm{X}\left(\mathrm{t}_{\mathrm{c}}\right)$.

Experimental points on Figure 11 represent the relative crystallinity of the four iPP samples at gel times $\mathrm{t}_{\mathrm{c}}$ for various degrees of undercooling $\Delta \mathrm{T}=10-26 \mathrm{~K}$. 


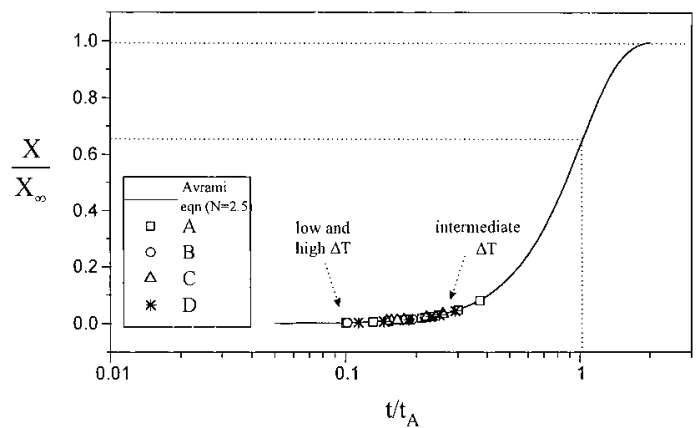

Figure 11. Relative crystallinity versus relative time $t / t_{A}$ according to eq 12 with the use of isothermal DSC data by Kim et al. (solid line). Points represent the relative crystallinity for the four iPP samples A-D at the relative times $t_{d} / t_{A}$ at which the critical gel state is reached at various undercoolings $\Delta \mathrm{T}=10-26 \mathrm{~K}$.

The plot clearly shows that gelation occurs very early in the crystallization process. The finding is in agreement with the observation of Schwittay et al. ${ }^{1}$ Nearly all of the crystallization process occurs on the template of the solid structure of the critical gel. The critical relative crystallinity $\mathrm{X}_{d} \mathrm{X}_{\infty}=\mathrm{X}\left(\mathrm{t}_{c}\right) / \mathrm{X}_{\infty}$ is extremely low for all samples, less than $3 \%$.

In Figure 11, the data are too close together to be resolved. For additional analysis, is necessary to show the effect of $\Delta T$ on $X_{c}$. The highest relative crystallinity is found for critical gels with intermediate undercooling where $X_{d} X_{\infty} \approx 2 \%$ (which corresponds to the absolute crystallinity $\left.\mathrm{X}_{\mathrm{c}} \approx 2 \% \times 0.6=1.2 \%\right)$ and $\mathrm{t}_{\mathrm{c}} \approx(0.3-0.4)$ $t_{A}$. Lower crystallinities, less than $1 \%$, are found in critical gels formed at both low and high undercooling $\Delta T$. The critical crystallinity goes through a maximum at intermediate $\Delta \mathrm{T}$. This will be analyzed next.

Linear Analysis of Critical Crystallinity $\mathbf{X}_{\mathbf{c}}$. Crystallization may be viewed as two ongoing processes. First is the radial growth of aggregates and fibrous "strands" governed by radial growth rate. This process is responsible for interconnected network formation (gelation) and is manifested experimentally in $t_{c}$. The second is internal crystallization inside the aggregates (through making them volume-filled with ordered crystals) governed by the internal crystallization rate. This process is responsible for the growth of crystallinity and is manifested experimentally through $t_{A}$. If the radial growth is dominating (high radial growth rate, short $t_{c}$ ), the crystallinity at GP will be lower (small values of $t_{d}$ $t_{A}$ ) and vice versa. The competition of these processes may be evaluated from the dimensionless group (division of eq 8 by eq 14)

$$
\frac{t_{c}}{t_{A}}=\frac{a}{A} e^{\Delta T(B-b)}
$$

Gel times $t_{c}$ and Avrami times $t_{A}$ were taken from monoexponential approximations eqs 8 and 14 with corresponding coefficients $a, b, A$, and $B$ listed in Table 2.

The decision on whether the critical crystallinity increases with $\Delta T$ rests on the difference of parameter values $(B-b)$, and the magnitude of $X_{c}$ depends on the ratio a/A. This can be seen when inserting eq 15 into eq 12:

$$
\frac{X_{c}}{X_{\infty}}=1-\exp \left\{-\left(\frac{a}{A}\right)^{N} \exp [N(B-b) \Delta T]\right\}
$$

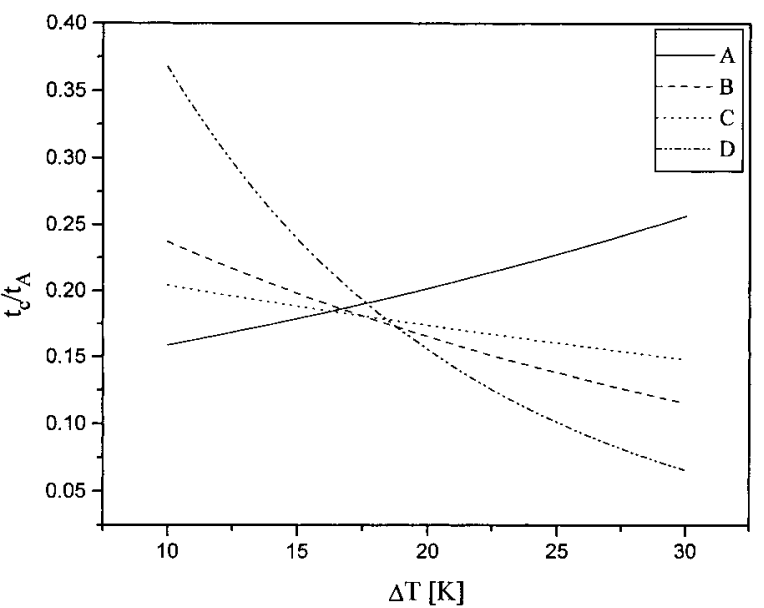

Figure 12. Characteristic time ratio at the gel point versus degree of undercooling for the four iPP samples. $t_{d} / t_{A}$ were estimated according to eq 15 with the use of a linear approximation for $\ln \left(t_{C}\right)$ and $\ln \left(t_{A}\right)$ and corresponding coefficients $\mathrm{a}, \mathrm{b}, \mathrm{A}$, and $\mathrm{B}$ (Table 2 ).

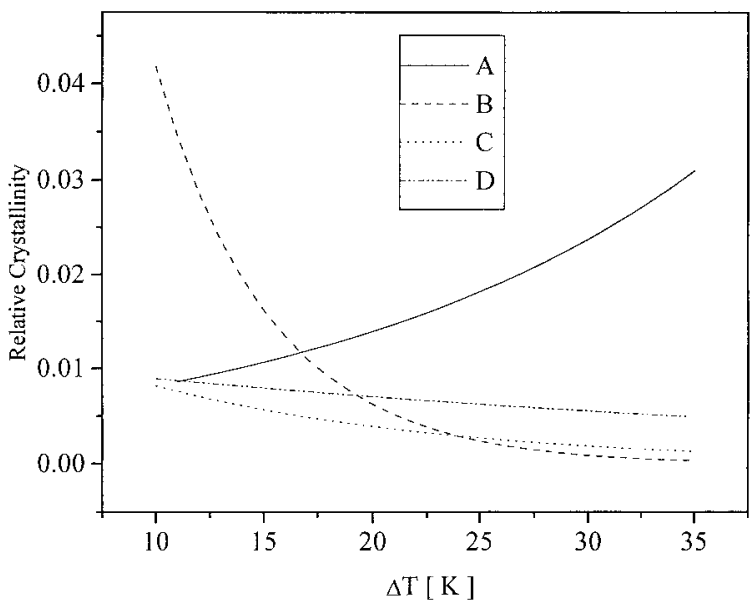

Figure 13. Relative crystallinity at the gel point $X_{d} / X_{\infty}$ versus degree of undercooling for the four iPP samples. $X_{\delta} X_{\infty}$ was estimated according to eqs 12 , and 14 with the use of linear approximation for $\ln \left(t_{c}\right)$ and $\ln \left(t_{A}\right)$ and corresponding coefficients $a, b, A$, and $B$ (Table 2).

To analyze the sensitivity to different undercooling $\Delta \mathrm{T}$, we differentiate eqs 15 and 16 with respect to $\Delta T$

$$
\frac{\partial}{\partial \Delta T}\left(\frac{t_{c}}{t_{A}}\right)=\left(\frac{A}{a}\right) \exp ^{(B-b) \Delta T}(B-b)
$$

$$
\begin{aligned}
& \frac{\partial}{\partial \Delta T}\left(\frac{X_{c}}{X_{\infty}}\right)=\left(\frac{a}{A}\right)^{N}(B-b) N e^{N(B-b) \Delta T} \times \\
& \exp \left\{-\left(\frac{a}{A}\right)^{N} e^{N(B-b) \Delta T}\right\}
\end{aligned}
$$

The highest sensitivity occurs with zero derivative of gel time $\partial / \partial \Delta T\left(t_{d} / t_{A}\right)=0$ and $\partial / \partial \Delta T\left(X_{d} / X_{\infty}\right)=0$. Both conditions lead to

$$
B-b=0 \quad \text { or } \quad \frac{B}{b}=1
$$

The critical crystallinity, $X_{C}$, increases with degree of undercooling, $\Delta T$, if $B / b>1$ (see sample $A$ in Figures 12 and 13). The opposite is found for samples $B, C$, and $D$ (see Figures 12 and 13) where $B / b<1$ and $X_{c}$ 


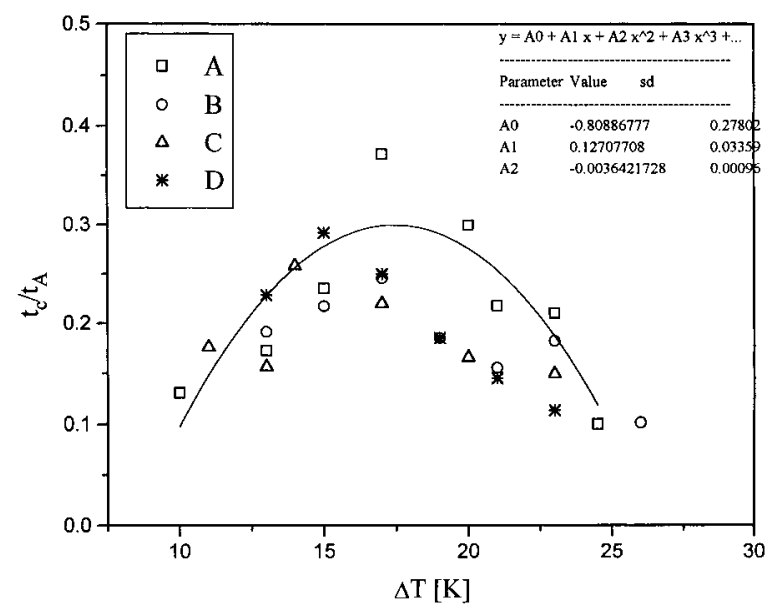

Figure 14. Characteristic time ratio at the gel point versus degree of undercooling for the four iPP samples (points). $t_{d} / t_{A}$ was estimated with the use of experimental gel times $t_{C}$ from Figure 9 and Avrami times $\mathrm{t}_{\mathrm{A}}=2.66 \times 10^{6} \mathrm{e}^{-0.224} \Delta \mathrm{T}$ from $\mathrm{Kim}$ et al. ${ }^{29}$ The solid line represents the fit by an empirical parabolic function $\mathrm{t}_{d} / \mathrm{t}_{\mathrm{A}}=-0.809+0.127(\Delta \mathrm{T})-0.0036(\Delta \mathrm{T})^{2}$ with a maximum at $\Delta \mathrm{T}=17.6 \mathrm{~K}$.

decreases with $\Delta \mathrm{T}$. In these samples, crystallization is very effective in generating long range connectivity.

Nonlinear Analysis of Experimental Data. Linearization of the temperature dependence of $t_{c}$ with a single-exponential function (eq 8) is only a crude approximation. Figure 9 unambiguously shows the downward concavity with constantly changing slope $b$ in eq 8 for each sample, which suggests a temperaturedependent rate of gelation and multiexponential nature of $\mathrm{t}_{\mathrm{c}}=\sum_{i} \mathrm{a}_{i}(\mathrm{~T}) \mathrm{e}^{-\mathrm{b}_{i}(\mathrm{~T}) \Delta \mathrm{T}}$. This means that with increasing undercooling, the slope $b$ of $t_{c}(\Delta T)$ is at first lower $(b<$ $B)$, then is equal $(b=B)$, and finally exceeds $(b>B)$ the slope of the Avrami time $t_{A}(\Delta T)$ (see Figure 9). We assume that slope $B$ of the Avrami time in eq 14 is constant as it follows from $\mathrm{Kim}$ et al. ${ }^{29}$ see the solid line in Figure 9. More data will be needed to explore the possibility that the rate of crystallinity growth might also be temperature dependent (and not constant in the whole tested temperature range). The characteristic time ratio $t_{d} t_{A}$ (Figure 14) and relative crystallinity $X_{d}$ $\mathrm{X}_{\infty}$ (Figure 15) go through a maximum at a degree of undercooling of about $\Delta \mathrm{T}=17 \mathrm{~K}$. Crystallization is most effective in producing connectivity for both small $\Delta \mathrm{T}$ and large $\Delta \mathrm{T}$.

It is interesting to relate the observations of $X_{c}(\Delta T)$ to the rheological parameters $S$ and $n$ of the critical gel. At low undercooling the network is "loose". It consists of a few non-volume-filled big spherulites formed by highly functional macromolecules that aggregate by their long sequences (segments) and span the entire sample. The gel is soft. High undercooling, in comparison, is known to increase the nucleation rate. At high nucleation density, the crystals do not have to grow much before they form a percolating structure. The network consists of a large number of small interconnected spherulites. The gel becomes stiffer. This is deduced from the simultaneous change in the material parameters: gel stiffness increases, and relaxation exponent decreases with undercooling (Figures 7 and 8).

The maximum in the crystallinity at intermediate undercooling $\Delta \mathrm{T}=18 \mathrm{~K}\left(\mathrm{~T}_{\exp }=145^{\circ} \mathrm{C}\right)$ corresponds to the maximum in crystallinity growth rate $t_{d} / t_{A}$. This suggests that gelation is unfavorable in this particular

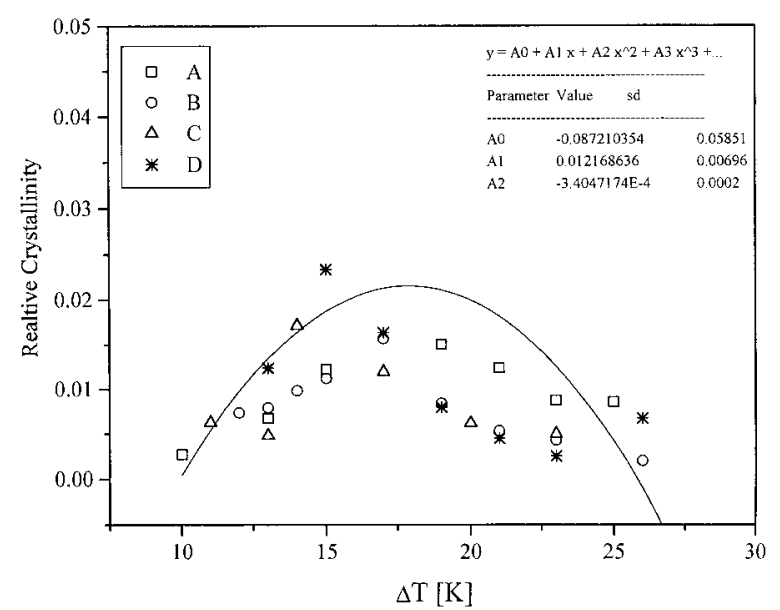

Figure 15. Relative crystallinity at the gel point $X_{d} X_{\infty}$ versus degree of undercooling for the four iPP samples (points). $X_{d}$ $\mathrm{X}_{\infty}$ was estimated according to eqs 12 , and 14 with the use of experimental gel times $t_{c}$ from Figure 9 and Avrami times $t_{A}$ $=2.66 \times 10^{6} \mathrm{e}^{-0.224} \Delta \mathrm{T}$ from $\mathrm{Kim}$ et al.29 The solid line represents the fit by an empirical parabolic function $X_{c}=$ $-0.087+0.012(\Delta \mathrm{T})-3.405 \times 10^{-4}(\Delta \mathrm{T})^{2}$ with a maximum at the same undercooling $\Delta \mathrm{T}=17.6 \mathrm{~K}$.

temperature region in comparison with crystallinity growth. We would think that this temperature-dependent maximum in crystallization rate and in relative crystallinity is not limited to iPP. It might be a general phenomenon for semicrystalline polymers that will need to be explored further with other polymers.

In iPP this temperature-dependent maximum may be due to the increase in the rate of ordered crystal branching, which was observed for this polymer in a certain temperature range. ${ }^{25-28}$ The molecular origin of this branching is the increasing interchain interaction as the molecular mobility slows down. In iPP these interactions may arise from methyl side groups, as the most spread out in space. Favorable interactions of side chains is realized when helix axes of the "parent" and "daughter" growing lamellae are oriented nearly perpendicular to each other. This specific relative chain orientation gives rise to highly ordered crystal branching and occurs at a specific crystallization temperature. The temperature should be relatively high to ensure slow growth rate, yet not high enough to preclude deposition of daughter lamellae. The preference for multidirectional (branching) or unidirectional (aggregation of long segments) growth in iPP is governed by a balance of intermolecular interactions and requires conformational energy calculations, which are out of our scope.

We based the interpretation of our experimental data on the classical picture of nucleation and growth in early stages of crystallization. An alternative model has been proposed recently (on the basis of SAXS and WAXD studies), it presumes that the polymer undergoes meltmelt spinodal decomposition prior to crystallization with selective aggregation of short sequencies of "straightened-out chains", which later assembles into the lamellae of the crystals. ${ }^{49}$ Obviously, it cannot be decided from rheological experiments whether spinodal decomposition is occurring or not.

Rheol ogy does not give any such structural information. It measures molecular mobility and changes in mobility during the gelation process. With the scientific data presently available, either one of the interpretations of early stages of crystallization (as a spinodal 
decomposition process and as nucleation and growth process) may be valid.

We have started experiments on the gelation of polyethylenes ${ }^{50}$ with various branching and found similar rheological patterns as in iPP. This indicates that there is some universal validity to our findings. Although crystallization in polyolefins of different molecular structures results in a wide variety of different morphologies (i.e., spherulites in polypropylenes, twisted lamellae stacks in polyethylene, etc.), the gel state with a characteristic "loose" network at early stages of crystallization and low undercooling is similar for all semicrystalline polymers.

\section{Conclusions}

The liquid/solid transition in crystallizing polypropylenes occurs at the very earliest stages of the crystallization process. The crystallinity is so low that it is hardly noticeable with other methods besides rheol ogy. The origin of the large-scale connectivity will need to be explored further since rheology only gives the dynamics of gelation and the mechanical properties at the gel point.

The highest absolute critical crystallinity $X_{c} \approx 2 \%$ was found at an intermediate undercooling, $\Delta T \approx 17 \mathrm{~K}$. This value seems to be about the same for all four samples. It depends on the activation energies for $t_{c}$ and $t_{A}$, as expressed in the exponents $b$ and $B$ in eqs 8 and 14 . Lower $X_{C}$ values were found at lower $\Delta T$ and at higher $\Delta T$. The highest $X_{c}$ occurs at $b / B=1$.

The stiffness of the critical gels increases with $\Delta T$ and the relaxation exponent adopts values of about 0.5 (which is very similar to the relaxation exponent of chemical critical gels from high molecular weight precursors ${ }^{37}$ ).

The solidification at the very earliest stages of crystallization of polymers has severe consequences for polymer processing, gelation is assumed to be the origin of the extreme sensitivity to small variations in molecular structure. Molecular architecture determines the crystallization kinetics and habit and, thus, the crystallization rate and the stiffness of the critical gel state.

In the future, flow effects on early stages of crystallization will need to be considered in order to apply the above concepts to polymer processing. In addition, scattering studies (SAXS, SALS) will be applied to monitor the structure development.

Acknowledgment. The financial support by a grant from ONR is gratefully acknowledged. We are grateful to Dr. Watson (Exxon Chemical, Baytown, TX) for providing GPC data.

\section{References and Notes}

(1) Schwittay, C.; Mours, M.; Winter, H. H. Faraday Discuss. 1995, 101, 93-104.

(2) te Nijenhuis, K.; Winter, H. H. Macromolecules 1989, 22, $411-414$

(3) te Nijenhuis, K. Adv. Polym. Sci. 1997, 130, 1-252.

(4) Lin, Y. G.; Mallin, D. T.; Chien, J. C. W.; Winter, H. H. Macromolecules 1991, 24, 850.

(5) Richtering, H. W.; Gagnon, K. D.; Lenz, R. W.; Fuller, R. C.; Winter, H. H. Macromol ecules 1992, 25, 2429-2433.

(6) Akpalu, I.; Hsiao, B. S.; Stein, R. S.; Muthukumar, M. Macromolecules, in press.

(7) Okada, T., Saito, H., Inoue, T. Macromolecules 1992, 25, $1908-1911$.

(8) Olley, R. H.; Bassett, D. C. Polymer 1989, 30, 399-409.

(9) Lauritzen, J . I.; Hoffman, J. D. J. Appl. Phys. 1973, 44, 4352.

(10) Hoffman, J. D. SPE Transactions. 1964, October, 315-362.
(11) Winter, H. H.; Chambon, F. J . J . Rheol. 1986, 30, 367-382.

(12) Chambon, F.; Winter, H. H. J . Rheol. 1987, 31, 683-697.

(13) Chambon, F.; Winter, H. H. Polym. Bull. 1985, 13, 499-503.

(14) Winter, H. H.; Mours, M. Adv. Polym. Sci. 1997, 134, 165234.

(15) Holly, E. E.; Venkataraman, S.; Chambon, F.; Winter, H. H. J . Non-Newtonian Fluid Mech. 1988, 27, 17-26.

(16) Schosseler, S.; Leibler, L. Phys. Lett. 1984, 45, 501.

(17) Stauffer, D. Pure Appl. Chem. 1981, 53, 1479.

(18) Stauffer, D. Introduction to Percolation Theory; Taylor and Francis: Philadel phia, PA, 1985.

(19) Falkai, B. v. Makromol. Chem. 1960, 41, 86-109.

(20) Frank H. P. Polypropylene; Gordon and Breach, New York, 1968; 134 pp.

(21) Eder, G.; J aneschitz-Kriegl, H.; Liedauer, S. Prog. Polym. Sci. 1990, 15, 629-714.

(22) Keith H. D.; Padden F. J . J . Appl. Phys. 1964, 35, 12701285, 1286-1296.

(23) Fatou, J. G. In Encyclopedia of Polymer Science and Engineering, Suppl. Vol.; J . Wiley: New York, 1989.

(24) Phillips, P. J . Rep. Prog. Phys. 1990, 53, 549-604.

(25) Padden, F. J ., J r.; Keith, H. D. J . Appl. Phys. 1973, 44, 12171223.

(26) Lovinger, A J . J . Polym. Sci., Part B 1983, 21, 97-110.

(27) Norton, D. R.; Keller, A. Polymer 1985, 26, 704-716.

(28) Lotz, B.; Wittmann, J . C. J . Polym. Sci., Part B 1986, 24, $1541-1558$

(29) Kim, C. Y.; Kim, Y. C.; Kim, S. C. Polym. Eng. Sci. 1993, 33, 1445-1451.

(30) Monasse, B.; Haudin, J. M. Colloid Polym. Sci. 1985, 263, 822-831.

(31) Fatou, J. G. Eur. Polym. J . 1971, 7, 1057-1064.

(32) Mours, M.; Winter, H. H. Rheol. Acta 1994, 33, 385-397.

(33) Ferry, J . D. Viscoelastic Properties of Polymers; Wiley: New York, 1980.

(34) Zeichner, G. R.; Patel, P. D. Proceedings of the 2nd World Congress of Chemical Engineering, Montreal, Quebec, Canada, Oct 4-9, 1981.

(35) Chambon, F. Proc. SPE Annu. Tech. Conf. Exhibits, 53rd 1995, 1157.

(36) Winter, H. H.; Morganelli, P.; Chambon, F. Macromolecules 1988, 21, 532-535.

(37) Mours, M.; Winter, H. H. Macromolecules 1996, 29, 72217229.

(38) Ziabicki, A. Fundamentals of Fibre Formation: the Science of Fibre Spinning and Drawing; Wiley: London, New York, 1976; $488 \mathrm{pp}$

(39) Ziabicki, A.; Kawai, H. High-Speed Fibre Spinning: Science and Enginering Aspects; Wiley: New York, 1985; 586 pp.

(40) Eder, G.; J aneschitz-Kriegl, H. Crystallization. From Processing of Polymers; VCH: Weinheim, 1997; Vol. 18, Chapter 5, p 269.

(41) Piccarolo, S. J . Macromol. Sci. 1992, B31 (4), 501.

(42) Piccarolo, S.; Saiu, M.; Brucato, V.; Titomanlio, G. J . Appl. Polym. Sci. 1992, 46, 625

(43) Scanlan, J . C.; Winter, H. H. Makromol. Chem., Macromol. Symp. 1991, 45, 11-21.

(44) Izuka, A.; Winter, H. H.; Hashimoto, T. Macromol ecules 1994 $27,6883-6888$

(45) I zuka, A.; Winter, H. H.; Hashimoto, T. Macromol ecules 1992, $25,5,2422-2428$.

(46) Avrami, M. J . Chem. Phys. 1939, 7, 1103-1112.

(47) Avrami, M. J . Chem. Phys. 1940, 8, 212-224.

(48) Avrami, M. J . Chem. Phys. 1941, 9, 177-184.

(49) Terrill, N.J .; Fairclough, P. A.; Towns-Andrews, E. T.; Ryan, A. Polym. Commun. 1998, 11, 2381.

(50) Gelfer, M.; Schmidt-Rohr, K.; Winter, H. H. in preparation. MA980134L 